\title{
Neuropsychological profile of Alzheimer's disease based on amyloid biomarker findings results from a South American cohort.
}

\author{
Autores \\ Maria Florencia Clarens, Lucia Crivelli , Ismael Calandri, Patricio Chrem Méndez, María Eugenia \\ Martin, María Julieta Russo, Jorge Campos, Ezequiel Surace, Silvia Vázquez, Gustavo \\ Sevlever, Ricardo Francisco Allegri.
}

\section{Abstract}

Objective: Increased life expectancy and exponential growth of adults suffering from Alzheimer's disease (AD) worldwide, has led to biomarkers incorporation for diagnosis in early stages. Use of neuropsychological testing remains limited. This study aimed to identify which neuropsychological tests best indicated underlying AD pathophysiology.Methods: One hundred and forty-one patients with $\mathrm{MCl}$ (Mild Cognitive Impairment) were studied. A neuropsychological test battery based on the Uniform Data Set (UDS) from the Alzheimer's Disease Centers program of the National Institute on Aging (NIA) was performed and amyloid markers recorded; according to presence or absence of amyloid identified by positive PIB-PET findings, or low CSF A 42 levels, patients were separated into $\mathrm{MCl}$ amyloid- $(n: 58)$ and $\mathrm{MCl}$ amyloid $+(n=83)$ cases. Results: Statistical differences were found in all memory tests between groups. Delayed recall score at thirty minutes on the Rey Auditory Verbal Learning Test (AVLT) was the best predictor of amyloid pathology presence (AUC 0.68), followed by AVLT total learning (AUC 0.66) and AVLT Recognition (AUC 0.59) scores, providing useful cut off values in the clinical setting.Conclusions: Use of neuropsychological testing, specifically AVLT scores with cutoff values, contributed to the correct diagnosis of $\mathrm{MCl}$ due to $A D$ in this SouthAmerican cohort.

\section{Palabras clave}

Ab42, Alzheimer's disease, cognitive biomarkers, dementia, mild cognitive impairment, neuropsychology. 